\title{
Zeldovich and the Missing Baryons, Results from Gravitational Lensing
}

\author{
Rudolph E. Schild \\ Harvard-Smithsonian Center for Astrophysics, \\ 60 Garden St, Cambridge, MA 02138, U.S.A.
}

\begin{abstract}
Central to Zeldovich's attempts to understand the origin of cosmological structure was his exploration of the fluid dynamical effects in the primordial gas, and how the baryonic dark matter formed. Unfortunately microlensing searches for condensed objects in the foreground of the Magellanic Clouds were flawed by the assumption that the objects would be uniformly (Gaussian) distributed, and because the cadence of daily observations strongly disfavored detection of planet mass microlenses. But quasar microlensing showed them to exist at planetary mass at the same time that a hydro-gravitational theory predicted the planet-mass population as fossils of turbulence at the time of recombination $(\mathrm{z}=1100$; Gibson 1996, 2001). Where the population has now been detected from MACHO searches to the LMC (Sumi et al. 2011) we compare the quasar microlensing results to the recent determination of the mass distribution function measured for the planetary mass function, and show that the population can account for the baryonic dark matter.
\end{abstract}

An important component of the Zeldovich sky is the baryonic dark matter, also called the missing baryons. Direct searches for them in the direction of the Galactic Bulge are hampered by the fact that objects smaller than Jupiter have Einstein rings that resolve the illuminated stellar disc of the background star being microlensed, producing the low efficiency of microlensing illustrated for Galactic Bulge backgrounds in Fig. 5 of Sumi et al. (2013). However, this is not a problem for searches with gravitational microlensing of quasars, since as discussed by Schild et al. (2012) earth mass and even lunar mass microlensing is observable for ordinary microlenses in the foreground galaxy G1 in the the double-image Q0957 gravitational lens system (The First Lens; Schild 1996).

A statistical analysis by Schild et al. (2012) of the microlensing statistics for Q0957+561 has shown that the mass function for planetary mass microlensing (nano-lensing) has a measured value of -2.98 , where the frequently observed Salpeter exponent is -2.35 . In other words, over the observed mass range in Schild (1999) the number of observed objects increases as approximately the third power of the reciprocal mass. Herein we will adopt a value of -3 for the exponent, which was estimated to be -2.98 with an error of $+/-0.5$.

These statistics will allow us to formulate a simple estimate of the amount of baryonic dark matter contained in the observed microlensing population, with just the assumption that the planetary range mass function in our Galaxy is the same value in quasar microlensing lens galaxy G1. In other words, since the population condensed as a primordial fog with droplet size approximately $1 M_{E \text { arth }}$ the primordial population was reasonably assumed to be a function of the baryonic density at time of recombination, and thus universal.

We begin our calculation by adopting the conclusion of Sumi et al. (2011) that in the solar neighborhood the number of Jupiter mass rogue planets equals approximately the number of stars. 
Adopting the approximation that the mass of Jupiter $M_{J}$ is 0.003 times the mass of the sun, $M_{\odot}$, we easily compute that the number of Earth-mass planets per star is a billion $(\times 30$ the estimate of Gibson 1996), and the mass of each is a millionth of the solar mass. Thus the baryonic mass in a population of rogue planets is 1000 solar masses, which is sufficient to account for the missing baryonic matter, (which has always been assumed to be approximately a factor 100 more than the luminous stellar matter). Thus the population of planetary mass objects seen in quasar microlensing can account for the entire missing baryonic matter.

Surveys looking for microlensing to stars in the Magellanic clouds were undertaken with a low (semi-weekly) cadence and have assumed that the particles are uniformly (Gaussian) distributed. This does not take into account the prediction from the hydrodynamics community that the objects will be clumped in proto-globular clusters (Jean's clusters, containing $10^{6} M_{\odot}$ and the higher cadence survey of Renault et al. (1998) did not use a standard color system to convert from measured colors on the instrumental system,and so do not convert directly to effective temperatures. Evidence for the population seen in microlensing of several other quasars has been given by Nieuwenhuizen et al. (2010), who also discuss radio and other observational detections.

\section{References}

Gibson, Carl H. 1996, Applied Mechanics Review, 49, 299. See also ArXiv:9904.260

Gibson, Carl H. 2001, arXiv:astro-ph/0110248

Nieuwenhuizen, T. , Schild, R., \& Gibson, Carl H. 2010, arXiv, 1011, 2530

Schild, R. 1996, ApJ, 464, 125

Schild, R. 1999, ApJ, 514, 598

Schild, R., Nieuwenhuizen, T., \& Gibson, Carl H. 2012, Physica Scripta, 151, 014082

Sumi, T. et al. 2011, Nature, 473, 349

Sumi, T. et al. 2013, ApJ, 778, 150 